\title{
Belgeo
}

Revue belge de géographie

Arpenter le monde/Travelling across the world

\section{Éditorial : Arpenter le monde}

\section{Stéphane Gomis et Mauricette Fournier}

\section{OpenEdition}

\section{Journals}

Édition électronique

URL : http://journals.openedition.org/belgeo/12930

DOI : 10.4000/belgeo.12930

ISSN : 2294-9135

\section{Éditeur :}

National Committee of Geography of Belgium, Société Royale Belge de Géographie

\section{Référence électronique}

Stéphane Gomis et Mauricette Fournier, «Éditorial : Arpenter le monde », Belgeo [En ligne], 2 | 2014 mis en ligne le 17 décembre 2014, consulté le 22 septembre 2020. URL : http:// journals.openedition.org/belgeo/12930; DOI : https://doi.org/10.4000/belgeo.12930

Ce document a été généré automatiquement le 22 septembre 2020.

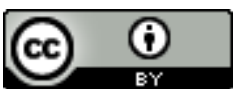

Belgeo est mis à disposition selon les termes de la licence Creative Commons Attribution 4.0 International. 


\title{
Éditorial : Arpenter le monde
}

\author{
Stéphane Gomis et Mauricette Fournier
}

1 Ce numéro thématique de la revue Belgeo, "Arpenter le monde ", s'inscrit dans le prolongement des travaux d'une équipe interdisciplinaire réunie à la Maison des Sciences de l'Homme de Clermont-Ferrand au sein du programme LIDO ( Des lieux, des œuvres. Représentations cartographiques, littéraires et iconographiques des lieux et des territoires ») qui s'articule autour de la problématique de la représentation des «lieux» (par la carte, l'image, les mots) et la dialectique des lieux et des œuvres (passage du lieu à l'œuvre et de l'œuvre au lieu). Nous souhaitions plus particulièrement saisir le lieu dans un contexte de mobilité et d'interculturalité afin d'appréhender comment l'œuvre restitue le lieu par le parcours physique et intellectuel des individus qui l'ont « arpenté » et comment, en retour, elle lui donne du sens. Cela nous a conduits à développer plus précisément la thématique du voyage et des voyageurs en l'associant de préférence à une réflexion sur les représentations cartographiques, domaine de recherches qui se caractérise actuellement par une grande vitalité dans de nombreuses disciplines. Les cartes cherchent à représenter le monde, ses lieux, ses territoires, ses paysages, pour mieux le « saisir ». Se faisant, elles révèlent l'imaginaire géographique des sociétés qui les conçoivent. Instrument d'interprétation du monde, elles constituent aussi un langage mobilisé tant pour essayer de rendre compte du réel (cartes documentaires des voyageurs, des géographes, des historiens) que de l'imaginaire (cartes et carnets de voyage d'artistes).

2 Les contributions rassemblées ici s'attachent à caractériser les facteurs qui marquent des évolutions essentielles dans la façon d'appréhender l'espace. Elles mettent en scène non pas des hommes qui seraient de simples témoins d'un monde en évolution mais bien des acteurs de ces bouleversements. Les thèmes de ces basculements de civilisations sont illustrés de façon diverse. Les auteurs se sont intéressés à différentes époques historiques depuis la fin de l'Antiquité jusqu'au XXI ${ }^{\mathrm{e}}$ siècle. De même, leurs terrains d'étude ne se limitent pas à l'Occident comme le montrent les apports concernant le continent africain ou les villes indiennes.

3 À l'échelle diachronique, l'Antiquité tardive est particulièrement sollicitée. Cet intérêt n'est pas surprenant. Période charnière entre Antiquité et Moyen Âge, elle s'avère 
essentielle afin de comprendre les évolutions en cours. Dans cette perspective, l'analyse conduite par Delphine Acolat éclaire d'un jour nouveau la perception des contemporains quant au franchissement des massifs de hautes montagnes. En l'espèce, les solutions proposées au IV siècle de notre ère par Ammien Marcellin, afin de répondre aux risques encourus par les voyageurs, sont particulièrement riches d'enseignements.

4 Pour leur part, Carlotta Franceschelli et Pier Luigi Dall'Aglio s'intéressent au changement des modalités du voyage entre Rome et Pavie au cours du $\mathrm{V}^{\mathrm{e}}$ siècle. Jusqu'alors essentiellement terrestre, ce périple sollicite la voie fluviale avec une navigation sur le Pô, pour relier Pavie à Ravenne. En outre, ils démontrent qu'en dehors des vestiges archéologiques, l'histoire des espaces et des axes de communication se nourrit aussi du témoignage de contemporains. En effet, il est à noter l'utilisation originale d'une source littéraire, en l'espèce la correspondance de Sidoine Apollinaire.

5 Les échanges épistolaires de celui-ci, tout à la fois homme politique et évêque, sont également au cœur de la contribution de Mauricette Fournier et Annick StoehrMonjou. Les évolutions du rapport à l'espace, au regard des bouleversements intervenus au cours du $\mathrm{V}^{\mathrm{e}}$ siècle, sont tout à fait perceptibles dans son œuvre. En l'espèce, la cartographie élaborée fournit un outil précieux d'interprétation du monde, qu'il soit réel ou imaginaire. L'approche à la fois géo-littéraire et géo-historique montre toute la richesse d'une démarche pluridisciplinaire.

6 Pour sa part, Fiona Lejosne nous fait entrer pleinement dans la modernité. Cela dit, la méthode mise en œuvre, afin d'éclairer les écrits du vénitien de la Renaissance G. B. Ramusio, revêt également une démarche interdisciplinaire en associant littérature et géographie historique. L'œuvre de cet auteur méconnu prend ainsi toute sa place parmi les travaux de savants confrontés à de nouvelles interprétations géopolitiques dans un monde en pleine expansion. Il est intéressant de voir combien Ramusio accorde toujours à la Sérénissime République une centralité, fut-elle symbolique.

$7 \mathrm{Si}$ les grandes découvertes révolutionnent la façon de représenter la Terre, la colonisation européenne du continent africain modifie à nouveau et profondément le rapport à l'espace. Jean-Michel Vasquez détaille les enjeux de l'appropriation territoriale en terre de mission. Le cas choisi est celui des voyages d'exploration, conduits au Zanguebar (Afrique orientale) dans les années 1877-1892, par un religieux spiritain le Père Alexandre Leroy. Son apostolat se double nécessairement d'une œuvre à la fois cartographique et ethnographique.

8 Les trois dernières contributions s'inscrivent résolument dans la période contemporaine. Pascale Argod retrace l'histoire du reportage graphique et propose un essai de définition et une typologie des carnets de voyage-reportage. Médium du témoignage du voyage vécu, l'art de l'illustration, grâce à sa richesse sémiotique, est à l'origine d'une grande diversité de genres (de l'album générique à la bande dessinée, du carnet documentaire au livre d'artiste, du roman graphique au photo-reportage de guerre, etc). Le carnet de voyage se caractérise dans tous les cas par une subjectivité assumée et une forte hybridité, annonciatrice de nouvelles formes d'art («art intermédia »).

9 Cette hybridité est illustrée par le travail de Bridget Sheridan. Plasticienne, cette dernière nous invite à la suivre sur le Chemin de la Liberté, une marche mémorielle de quatre jours qui conduit, à travers les Pyrénées ariégeoises, de Saint-Girons à Esterri d'Àneu en Espagne dans les pas des contrebandiers; puis des passeurs qui, pendant la 
Seconde Guerre Mondiale, ont permis à de nombreuses personnes de fuir le nazisme et le Service du Travail Obligatoire. L'expérience qu'elle relate permet d'appréhender le processus créatif qu'elle a mis en œuvre pour réaliser deux travaux artistiques, un carnet de marche et un foulard en soie - une Escape Map - sur lequel elle a cousu son trajet avec un fil rouge, symbolisant ainsi tant les traces de la mémoire collective que le maillage des relations sociales.

Les objectifs et la démarche de Carole Lanoix se révèlent assez similaires. S'appuyant sur l'observation in situ et la pratique de la déambulation pédestre, elle cherche à rendre compte de la complexité du lieu - en l'occurrence celle des villes indiennes - par des carnets de terrain qui se présentent comme des "carto-ethnographies " révélant des traces et des récits. Toutefois, à la différence des artistes auxquels elle emprunte partiellement les méthodes, ces carnets qui concentrent, dans la tradition des géographes et des anthropologues, les observations empiriques du chercheur, ne correspondent pas à un aboutissement, mais constituent au contraire un outil pour interroger la notion même de terrain. Arpenter le monde pour relever ses "petits riens » qu'il s'agira ensuite d'interpréter constitue aussi une méthode scientifique à réexpérimenter.

11 La thématique centrale de ce numéro est toute entière tournée sur le rôle et l'histoire des représentations associées à des lieux, la singularité des trajectoires spatiotemporelles. L'imaginaire est référencé à des types d'espaces, reconstruits par la perception de ceux qui les ont arpentés, ces «transcripteurs » influencés par leur culture, qu'ils soient voyageurs, missionnaires, cartographes, artistes ou chercheurs. La posture adoptée par ces acteurs comme leur qualité déterminent leur vision du monde, en dessinent les contours. Ils témoignent aussi des transformations des sociétés, de leurs capacités d'adaptation tout comme de l'importance d'une part des réseaux et maillages matériels (routes terrestres, maritimes...) comme immatériels (liens sociaux), d'autre part de la persistance des manifestations mémorielles.

\section{AUTEURS}

\section{STÉPHANE GOMIS}

Université Blaise Pascal - Clermont II, CHEC, s.gomis@neuf.fr

\section{MAURICETTE FOURNIER}

Centre d'Études et de Recherches Appliquées au Massif Central (CERAMAC), Université Blaise

Pascal - Clermont-Ferrand II, mf.43sauzet@orange.fr 